\title{
Nodularity-like appearance in the cardia: novel endoscopic findings for Helicobacter pylori infection
}

\section{다(1) $(\Theta$}

\section{Authors}

Osamu Toyoshima ${ }^{1,2}$, Toshihiro Nishizawa ${ }^{1,3}$, Kosuke Sakitani ${ }^{1,4}$, Tadahiro Yamakawa1, Hidenobu Watanabe ${ }^{5}$, Shuntaro Yoshida ${ }^{1,2}$, Yousuke Nakai ${ }^{2}$, Keisuke Hata ${ }^{1,6}$, Hirotoshi Ebinuma ${ }^{3}$, Hidekazu Suzuki ${ }^{7}$, Kazuhiko Koike

Institutions

1 Gastroenterology, Toyoshima Endoscopy Clinic, Tokyo, Japan

2 Department of Gastroenterology, Graduate School of Medicine, The University of Tokyo, Tokyo, Japan

3 Department of Gastroenterology and Hepatology, International University of Health and Welfare, Mita Hospital, Tokyo, Japan

4 Gastroenterology, Sakitani Endoscopy Clinic, Narashino, Japan

5 Pathology and Cytology Laboratory Japan, Tokyo, Japan

6 Department of Surgical Oncology, Graduate school of Medicine, The University of Tokyo, Tokyo, Japan

7 Department of Gastroenterology and Hepatology, Tokai University School of Medicine, Kanagawa, Japan

submitted 8.10.2019

accepted after revision 12.2 .2020

\section{Bibliography}

DOI https://doi.org/10.1055/a-1136-9890 |

Endoscopy International Open 2020; 08: E770-E774

(c) Georg Thieme Verlag KG Stuttgart · New York

elSSN 2196-9736

Corresponding author

Osamu Toyoshima, MD, Director, Gastroenterology,

Toyoshima Endoscopy Clinic, 6-17-5 Seijo, Setagaya-ku,

Tokyo 157-0066, Japan

Fax: +81-3-5429-9511

t@ichou.com

\section{ABSTRACT}

Background and study aims Helicobacter pylori-associated nodular gastritis, which is associated with follicular lymphoid hyperplasia, is mainly recognized in the antrum. However, we have also observed nodularity-like appearance in the cardia. This study aimed to investigate the clinical significance of cardiac nodularity-like appearance in $\mathrm{H}$. pylori-associated gastritis.

Patients and methods Patients who underwent esophagogastroduodenoscopy and were evaluated for H. pylori infection for the first time were enrolled. A nodularity-like appearance in the cardia was defined as a miliary nodular appearance or scattered appearances of small circular whitish coloration. $H$. pylori infection was diagnosed according to serum anti- $H$. pylori antibody and the urea breath test or histology. Accuracy of the $H$. pylori infection diagnoses based on nodularity-like appearance were assessed.

Results Among 265 patients, 42 patients (15.8\%) were diagnosed as positive for $\mathrm{H}$. pylori. Cardiac nodularity-like appearance and antral nodularity were recognized in 25 and 15 patients, respectively. In accuracy of predicting $H$. pylori by cardiac nodularity-like appearance, specificity was 0.996 , sensitivity was 0.571 , positive predictive value was 0.960 , negative predictive value was 0.925 , and accuracy was 0.928 . The sensitivity of cardiac nodularity-like appearance was significantly higher than that of antral nodularity $(P=0.0284)$.

Conclusions Cardiac nodularity-like appearance had a high accuracy rate for $H$. pylori infection diagnosis. Cardiac nodularity-like appearance was found more frequently than antral nodularity.

\section{Introduction}

Helicobacter pylori (H. pylori) infection is one of the most prevalent infectious diseases worldwide, with $40 \%$ to $50 \%$ of the global human population estimated to be infected [1]. H. pylori is associated with development of atrophic gastritis and gastric cancer [2-5], and eradication of $H$. pylori infection has been reported as an effective strategy for treating atrophic gastritis and peptic ulcer and preventing gastric cancer [6-9]. There- fore, it is important to estimate $H$. pylori infection status [10]. In chronic gastritis, endoscopic images of gastric mucosa display different characteristics according to the severity and duration of $H$. pylori infection. Endoscopic findings of diffuse redness, enlarged folds, nodularity, atrophy, and intestinal metaplasia are associated with chronic gastric mucosal inflammation and $H$. pylori infection [11]. Nodular gastritis is characterized by a miliary pattern resembling "gooseflesh" in the antral and/or corpus mucosa on endoscopy [12]. Nodular gastritis is 


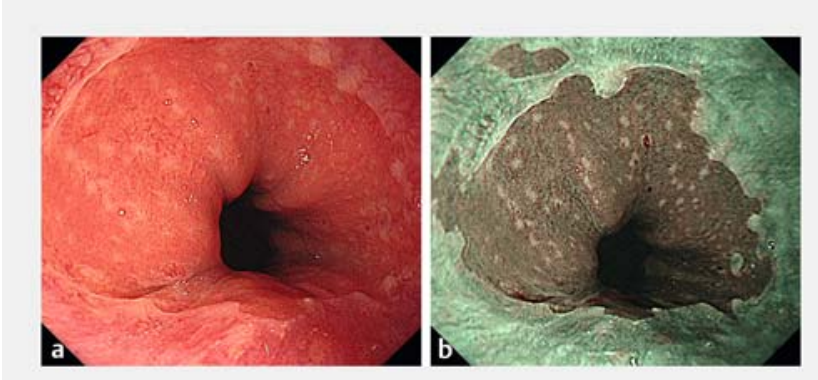

- Fig. 1 Endoscopic images of cardiac nodularity from a 48-yearold woman with $\mathrm{H}$. pylori antibody levels of $9.4 \mathrm{U} / \mathrm{mL}$, urea breath test of 28.5 permil, and antral nodularity. a White light observation. A miliary pattern resembling "gooseflesh": was found in the cardia. Whitish circular micronodules measuring $\leq 1 \mathrm{~mm}$ in both diameter and height were observed. b Narrow-band imaging observation. Whitish coloration denoted nodularity.

frequently associated with follicular lymphoid hyperplasia with intraepithelial lymphocytosis [13]. Nodularity can be seen in the stomach of children much more frequently than in adults and may be a characteristic of an early stage of infection with H. pylori [14]. Nodularity appears more commonly in the antral mucosa than in the corpus mucosa [13]. Recently, we have observed nodularity not only in the antrum but also in the cardia. Nodularity-like appearance in the cardia has never been evaluated; therefore, the aim of the current study was to investigate the clinical significance of cardiac nodularity-like appearance in $\mathrm{H}$. pylori-associated gastritis.

\section{Patients and methods}

\section{Ethics}

This study was approved by the ethical review committee of Hattori Clinic on September 6, 2019 (approval no. S1909-U06). Written informed consent was obtained from all participants $[5,15]$, and all clinical investigations were conducted according to the ethical guidelines of the Declaration of Helsinki.

\section{Patients}

Consecutive patients who underwent esophagogastroduodenoscopy (EGD) and a serum anti-H. pylori antibody test between July 2017 and July 2019 in the Toyoshima Endoscopy Clinic were retrospectively reviewed. We included patients who were evaluated for $H$. pylori infection for the first time and excluded patients with a history of eradication treatment, gastric cancer, or gastrectomy. EGD was conducted for screening and the examination of symptoms. Data on patient baseline characteristics, including age, sex, and indication for EGD, were collected.

\section{Endoscopic and pathological procedures}

EGD was performed using the Olympus Evis Lucera Elite system with a GIF-HQ290 or GIF-H290Z endoscope (Olympus Corporation, Tokyo, Japan) by an expert physician (O.T.). Furthermore, EGD images were retrospectively reviewed by other expert physicians. Discrepancies in diagnosis between the two sets of phy- sicians were resolved through discussion. Sedation with midazolam and/or pethidine was performed at patient discretion $[5,16]$. The diagnosis of nodularity in the antrum was made when the mucosa had a miliary nodular appearance. Characteristic findings were whitish circular micronodules measuring $\leq 1$ $\mathrm{mm}$ in both diameter and height. A nodularity-like appearance in the cardia was defined as a miliary nodular appearance or the presence of scattered whitish circular small colorations (flat type) within $2 \mathrm{~cm}$ of the esophagogastric junction. Nodularity was visualized as whitish in narrow-band imaging mode. Representative endoscopic images are shown in > Fig. 1.

We carried out targeted biopsy of the cardiac nodularity-like appearance. Pathological findings were evaluated using hematoxylin and eosin stain, and histological diagnosis was made by an expert gastrointestinal pathologist (H.W.).

We evaluated endoscopic gastric atrophy according to Kimura-Takemoto classification [17]. Widely spreading atrophy, where the border of the gastric lesser curvature extends beyond the cardia is defined as open-type atrophy.

\section{Diagnosis of $H$. pylori infection}

Serum anti-H. pylori antibody was measured on the day of EGD. The antibody titer was measured using an enzyme immunoassay kit with antigens derived from Japanese individuals (E-plate Eiken H. pylori antibody II; Eiken Chemical, Tokyo, Japan). The manufacturer recommended a cut-off value of $10 \mathrm{U} / \mathrm{mL}$ for $H$. pylori positivity. When the serum anti-H. pylori antibody titer was $\geq 3.0 \mathrm{U} / \mathrm{mL}$, the urea breath test or histology was added. If either the urea breath test or histology was positive, patients were considered positive for $H$. pylori $[18,19]$. An antibody titer $<3.0 \mathrm{U} / \mathrm{mL}$ or negative urea breath test was considered to indicate $H$. pylori negativity.

\section{Statistical analysis}

Accuracy of $H$. pylori infection diagnoses based on cardiac nodularity-like appearance and antral nodularity was assessed. To validate the nodularity-like appearance in the cardia, the interobserver agreement between 2 endoscopists was examined, and the kappa value was calculated. The association between nodularity and clinicopathological factors was analyzed. Categorical and continuous data were compared using the chisquare test and Mann-Whitney $U$ test, respectively. A two-sided $P<0.05$ was considered statistically significant. Data were analyzed using Ekuseru-Toukei 2015 software (Social Survey Research Information, Tokyo, Japan).

\section{Results}

The endoscopist performed 1332 EGDs during the study period. We excluded 523 patients with known $H$. pylori infection status ( 7 positive and 516 negative) and 534 patients after H. pylori eradication treatment. Two hundred and sixty-five patients were finally enrolled, after excluding 10 patients whose H. pylori infection status could not be identified ( $\mathbf{F i g} \mathbf{2}$ ).

Characteristics of the participants in the current study are shown in Table 1. A total of 42 patients (15.8\%) were diagnosed as positive for $H$. pylori. Cardiac nodularity-like appear- 


\section{2 underwent esophagogastroduodenoscopy}

523 with known $H$. pylori status

534 after $H$. pylori eradication

\section{5 with unknown $H$. pylori status}

10 with unidentified $H$. pylori status

265 eligible for the analysis

Fig. 2 Flowchart of patients.

- Table 1 Characteristics of enrolled subjects.

Total, n

Mean age, years (range)

Male sex, $n$

H. pylori (+), $\mathrm{n}$

Cardiac nodularity, $\mathrm{n}$

Antral nodularity, $\mathrm{n}$

Table 2 Prediction of $H$. pylori infection by cardiac nodularity.

\begin{tabular}{|l|l|c|}
\hline & H. pylori (+) & H. pylori (-) \\
\hline Cardiac nodularity (+) & 24 & 1 \\
\hline Cardiac nodularity (-) & 18 & 222 \\
\hline $\begin{array}{l}\text { Sensitivity } 0.571 \text {, specificity } 0.996, \text { positive predictive } \\
\text { value } 0.960, \text { negative predictive value } 0.925 \text {, accuracy } 0.928 .\end{array}$
\end{tabular}

- Table 3 Prediction of $H$. pylori infection by antral nodularity.

\begin{tabular}{|l|c|c|}
\hline & H. pylori (+) & H. pylori (-) \\
\hline Antral nodularity (+) & 14 & 1 \\
\hline Antral nodularity (-) & 28 & 222 \\
\hline $\begin{array}{l}\text { Sensitivity } 0.333, \text { specificity } \\
\text { value } 0.933, \text { negative predictive value } 0.888 \text {, accuracy } 0.891 .\end{array}$ \\
\hline
\end{tabular}

ance and antral nodularity were recognized in 25 and 15 patients, respectively. Gastric cancer was not found in the enrolled patients.

With cardiac nodularity-like appearance, accuracy of prediction of $H$. pylori infection is shown in $>$ Table 2 . Specificity was 0.996 (95\% confidence interval: $0.975-1.00$ ), the sensitivity was $0.571(0.410-0.723)$, the positive predictive value (PPV) was 0.960 (0.797-0.999), negative predictive value (NPV) was 0.925 (0.884-0.955), and the accuracy was 0.928 (0.890$0.956)$. The $\mathrm{k}$ value validating the interobserver agreement on the nodularity-like appearance in the cardia was excellent $(\mathrm{K}$ value=0.94).

With antral nodularity, accuracy of prediction of $\mathrm{H}$. pylori infection is shown in $>$ Table 3 . Specificity was 0.996 (95\% confidence interval: $0.975-1.00)$, sensitivity was $0.333(0.196-$ $0.496)$, PPV was 0.933 (0.681-0.998), NPV was 0.888 (0.842$0.924)$, and accuracy was 0.891 (0.847-0.926). Cardiac nodularity was observed in all patients with $H$. pylori infection with antral nodularity. Sensitivity of cardiac nodularity was significantly higher than that of antral nodularity $(P=0.0284)$. The $\mathrm{K}$ value validating the interobserver agreement on the antral nodularity was good $(\kappa$ value $=0.76)$.

Both the cardiac nodularity-like appearance and antral nodularity were associated with endoscopic open-type atrophy, but not with age or sex ( $\triangleright$ Table 4$)$.

Histological examination of cardiac nodularity was performed in 10 patients. Six of 10 specimens obtained from cardiac nodularity revealed lymphoid follicles displaying lymphocyte infiltration in the cardiac gland. Representative pathological images are shown in > Fig. 3.

\section{Discussion}

This is the first report to describe characteristics of cardiac nodularity-like appearance. Cardiac nodularity-like appearance was associated with $H$. pylori infection and had excellent accuracy for $H$. pylori infection diagnosis. In addition, sensitivity of cardiac nodularity-like appearance was significantly higher than that of antral nodularity. These results suggest that presence of cardiac nodularity-like appearance on EGD may be one of the predictors of $H$. pylori infection.

Reported incidence of this nodularity varies considerably and has been reported at $32.9 \%$ to $85 \%$ in $\mathrm{H}$. pylori-positive children [20-24]. Nodularity was previously considered to be a

- Table 4 Association between nodularity and clinicopathological factors.

\begin{tabular}{|l|l|l|l|l|r|r|}
\hline & $\begin{array}{l}\text { Cardiac nodularity } \\
(+)\end{array}$ & $\begin{array}{l}\text { Cardiac nodularity } \\
(-)\end{array}$ & P value & \multicolumn{2}{|c|}{$\begin{array}{l}\text { Antral nodularity } \\
(+)\end{array}$} & $\begin{array}{l}\text { Antral nodularity } \\
(-)\end{array}$ \\
\hline Number & 25 & 240 & & 15 & 250 \\
\hline Age, years & 46.2 & 48.5 & 0.41 & 48.2 & 48.3 \\
\hline Sex, male/female & $9 / 16$ & $103 / 137$ & 0.51 & $4 / 11$ & $108 / 142$ \\
\hline Atrophy, open/closed & $12 / 13$ & $16 / 224$ & $<0.001$ & $7 / 8$ & $21 / 229$ \\
\hline
\end{tabular}




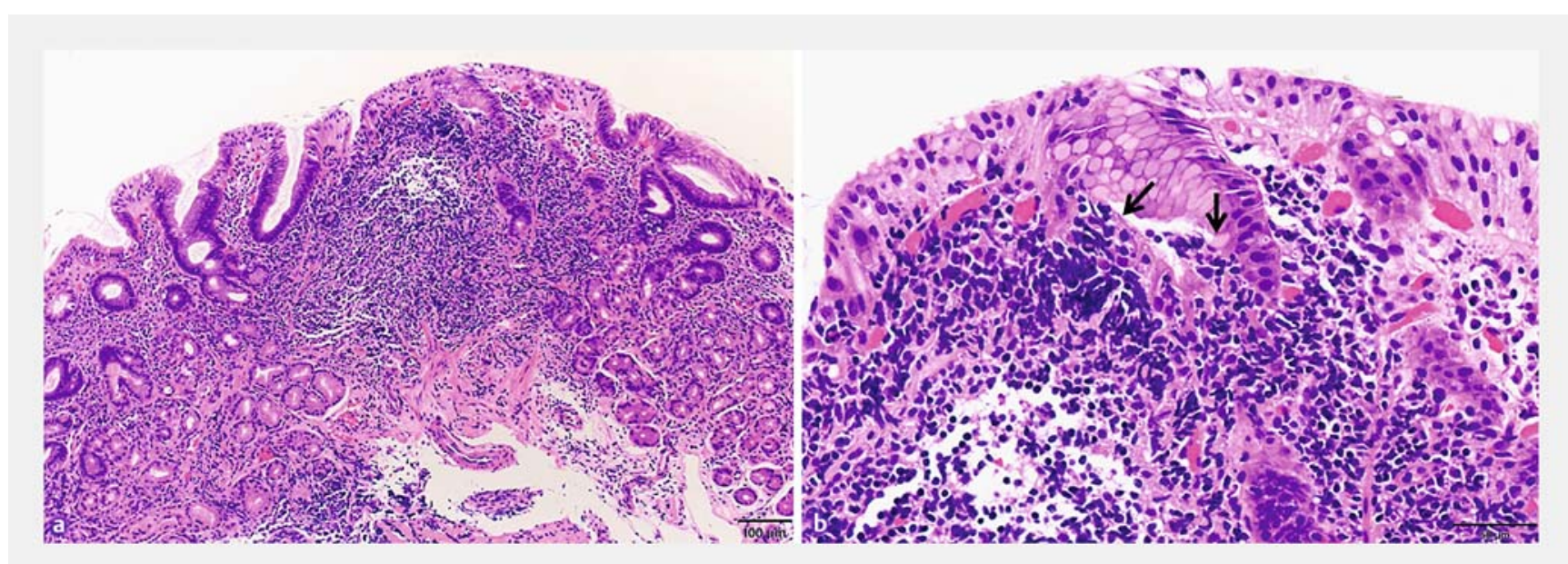

- Fig. 3 Pathological images of cardiac nodularity from a 48-year-old woman with $\mathrm{H}$. pylori antibody levels of $9.4 \mathrm{U} / \mathrm{mL}$, urea breath test result of 28.5 permil, and antral nodularity. a A lymphoid follicle was observed in the cardiac mucosa. The black scale indicates $100 \mu \mathrm{m}$. H\&E stain. b Intraepithelial lymphocyte infiltration was observed (arrows). The black scale indicates $50 \mu \mathrm{m}$. H\&E stain.

specific finding in children with $H$. pylori infections. However, recent reports indicated that nodularity is occasionally observed in adults with $H$. pylori infections, particularly in women and young adults [25]. Nodular gastritis improves gradually with age [22]. Antral nodularity corresponds to germinal follicles and lymphocytic aggregates in the gastric mucosa [13, 26]. In this study, we often found lymphoid follicles in the biopsy samples of the cardiac nodularity-like appearance. Nodular gastritis is associated with increased risk of developing diffusetype cancer due to highly active gastric inflammation. Nishikawa et al. reported that $H$. pylori eradication in patients with nodular gastritis effectively prevented diffuse-type cancer. Detection of nodular gastritis is important to prevent gastric cancer [14]. Further studies should be conducted to analyze the clinicopathological significance of cardiac nodularity-like appearance.

There are two types of inflammation at the gastric cardiac region: gastroesophageal reflux disease-related type and $H$. $p y$ lori-related type $[27,28]$. The cardiac nodularity-like appearance in this study might apply to $H$. pylori-related type carditis. Egi et al. reported that cardiac cancer was associated with $H$. pylori infection, and carditis was observed in all cardiac cancer patients with $H$. pylori infection $[29,30]$. Careful observation of cardia is recommended.

Lymphoid follicles are often observed in the antrum but less frequent in the fundic gland mucosa [13,31]. Eidt et al. reported that prevalence of lymphoid follicles in $H$. pylori gastritis was $54 \%$ in the antral and $15 \%$ in the corpus mucosa, respectively [31]. Furthermore, the corpus mucosa is so thick that it could hinder the endoscopic observation [32]. Therefore, the reason why nodularity is rarely observed in the corpus may be due to the low prevalence of lymphoid follicles and thick mucosa in the corpus. In this study, lymphoid follicles were often recognized in the cardia. The cardiac and pyloric glands are mainly responsible for mucus secretion and bicarbonate secretion and act as a defense mechanism for gastric mucosa. Both express
MUC6 and secrete pepsinogen II, but not pepsinogen I. Fundic glands are composed of chief cells (secreting both pepsinogen I and II), parietal cells (acid secretion), and mucus neck cells (mucus secretion), and are responsible for gastric secretion related to gastric digestion [33]. Similarities of cardia and pyloric glands, unlike fundic glands, might contribute to the appearance of lymphoid follicles in the cardia.

This study had some limitations. First, it was a retrospective review at a single institution. Second, it employed only a single experienced endoscopist. Therefore, it is difficult to apply these findings directly to other endoscopists. Third, Cag A status was not assessed [34]. Further analyses of other bacterial factors, along with host genetic and environmental factors that modulate the response to $H$. pylori infection, are warranted. Fourth, the number of biopsy samples obtained from the nodularitylike appearance was too small to explain the histological feature of these endoscopic findings. In the future, further analysis is needed.

\section{Conclusion}

In conclusion, $H$. pylori-associated nodularity may appear in the cardia. Cardiac nodularity-like appearance was found more frequently than antral nodularity in this study.

\section{Acknowledgement}

The authors thank Editage (www.editage.com) for English language editing.

\section{Competing interests}

The authors declare that they have no conflict of interest. 


\section{References}

[1] Suzuki H, Nishizawa T, Hibi T. Helicobacter pylori eradication therapy. Future Microbiol 2010; 5: 639-648

[2] Suzuki H, Nishizawa T, Tsugawa $\mathrm{H}$ et al. Roles of oxidative stress in stomach disorders. J Clin Biochem Nutr 2012; 50: 35-39

[3] Nishizawa T, Suzuki H, Sakitani K et al. Family history is an independent risk factor for the progression of gastric atrophy among patients with Helicobacter pylori infection. United European Gastroenterol J 2017; 5: 32-36

[4] Toyoshima O, Tanikawa C, Yamamoto R et al. Decrease in PSCA expression caused by Helicobacter pylori infection may promote progression to severe gastritis. Oncotarget 2018; 9: 3936-3945

[5] Sakitani K, Nishizawa T, Arita M et al. Early detection of gastric cancer after Helicobacter pylori eradication due to endoscopic surveillance. Helicobacter 2018; 23: e12503

[6] Pimentel-Nunes P, Libânio D, Marcos-Pinto R et al. Management of epithelial precancerous conditions and lesions in the stomach (MAPS II): European Society of Gastrointestinal Endoscopy (ESGE), European Helicobacter and Microbiota Study Group (EHMSG), European Society of Pathology (ESP), and Sociedade Portuguesa de Endoscopia Digestiva (SPED) guideline update 2019. Endoscopy 2019; 51: 365-388

[7] Dinis-Ribeiro M, Areia M, de Vries AC et al. Management of precancerous conditions and lesions in the stomach (MAPS): guideline from the European Society of Gastrointestinal Endoscopy (ESGE), European Helicobacter Study Group (EHSG), European Society of Pathology (ESP), and the Sociedade Portuguesa de Endoscopia Digestiva (SPED). Endoscopy 2012; 44: 74-94

[8] Suzuki H, Nishizawa T, Tsugawa $H$ et al. Molecular approaches and modern clinical strategies for the management of Helicobacter pylori infection in Japan. Keio J Med 2012; 61: 109-119

[9] Toyoshima O, Yamaji Y, Yoshida S et al. Endoscopic gastric atrophy is strongly associated with gastric cancer development after Helicobacter pylori eradication. Surg Endosc 2017; 31: 2140-2148

[10] Itoh T, Kawahira H, Nakashima $\mathrm{H}$ et al. Deep learning analyzes Helicobacter pylori infection by upper gastrointestinal endoscopy images. Endosc Int Open 2018; 6: E139-e144

[11] Toyoshima O, Nishizawa T, Sakitani K et al. Serum anti-Helicobacter pylori antibody titer and its association with gastric nodularity, atrophy, and age: A cross-sectional study. World J Gastroenterol 2018; 24 : 4061-4068

[12] Miyamoto M, Haruma K, Yoshihara M et al. Nodular gastritis in adults is caused by Helicobacter pylori infection. Dig Dis Sci 2003; 48: 968975

[13] Hayashi S, Imamura J, Kimura K et al. Endoscopic features of lymphoid follicles in Helicobacter pylori-associated chronic gastritis. Dig Endosc 2015; 27: 53-60

[14] Nishikawa I, Kato J, Terasoma $\mathrm{S}$ et al. Nodular gastritis in association with gastric cancer development before and after Helicobacter pylori eradication. JGH Open 2018; 2: 80-86

[15] Nishizawa T, Suzuki H, Arano T et al. Characteristics of gastric cancer detected within 1 year after successful eradication of Helicobacter pylori. J Clin Biochem Nutr 2016; 59: 226-230

[16] Toyoshima O, Yoshida S, Nishizawa T et al. CF290 for pancolonic chromoendoscopy improved sessile serrated polyp detection and procedure time: a propensity score-matching study. Endosc Int Open 2019; 7: E987-E993

[17] Kimura K, Takemoto T. An endoscopic recognition of the atrophic border and its significance in chronic gastritis. Endoscopy 1969; 3 : $87-97$

[18] Toyoshima O, Nishizawa T, Arita M et al. Helicobacter pylori infection in subjects negative for high titer serum antibody. World J Gastroenterol 2018; 24: 1419-1428

[19] Nishizawa T, Sakitani K, Suzuki H et al. A combination of serum antiHelicobacter pylori antibody titer and Kyoto classification score could provide a more accurate diagnosis of $\mathrm{H}$ pylori. United European Gastroenterol J 2019; 7: 343-348

[20] Bujanover Y, Konikoff F, Baratz M. Nodular gastritis and Helicobacter pylori. J Pediatr Gastroenterol Nutr 1990; 11: 41-44

[21] Mitchell HM, Bohane TD, Tobias V et al. Helicobacter pylori infection in children: potential clues to pathogenesis. J Pediatr Gastroenterol Nutr 1993; 16: 120-125

[22] Shiotani A, Kamada T, Kumamoto M et al. Nodular gastritis in Japanese young adults: endoscopic and histological observations. J Gastroenterol 2007; 42: 610-615

[23] Prieto G, Polanco I, Larrauri J et al. Helicobacter pylori infection in children: clinical, endoscopic, and histologic correlations. J Pediatr Gastroenterol Nutr 1992; 14: 420-425

[24] Luzza F, Pensabene L, Imeneo M et al. Antral nodularity and positive CagA serology are distinct and relevant markers of severe gastric inflammation in children with Helicobacter pylori infection. Helicobacter 2002; 7: 46-52

[25] Sbeih F, Abdullah A, Sullivan S et al. Antral nodularity, gastric lymphoid hyperplasia, and Helicobacter pylori in adults. J Clin Gastroenterol 1996; 22: 227-230

[26] Zerbib F, Vialette G, Cayla R et al. Follicular gastritis in adults. Relations with Helicobacter pylori, histological and endoscopic aspects. Gastroenterol Clin Biol 1993; 17: 529-534

[27] Cestari R, Villanacci V, Bassotti G et al. The pathology of gastric cardia: a prospective, endoscopic, and morphologic study. Am J Surg Pathol 2007; 31: 706-710

[28] Uedo N, Yoshio T, Yoshinaga S et al. Endoscopic gastric mucosal atrophy distinguishes the characteristics of superficial esophagogastric junction adenocarcinoma. Dig Endosc 2017; 29: 26-36

[29] Egi Y, Kim S, Ito M et al. Helicobacter pylori infection is the major risk factor for gastric inflammation in the cardia. Dig Dis Sci 2006; 51: 1582-1588

[30] Egi Y, Ito M, Tanaka S et al. Role of Helicobacter pylori infection and chronic inflammation in gastric cancer in the cardia. Jpn J Clin Oncol 2007; 37: 365-369

[31] Eidt S, Stolte M. Prevalence of lymphoid follicles and aggregates in Helicobacter pylori gastritis in antral and body mucosa. J Clin Pathol 1993; 46: 832-835

[32] Kim YJ, Lee SY, Lee SP et al. Identification of Nodular Gastritis among Patients Diagnosed with Lymphofollicular Gastritis on a Gastric Biopsied Specimen. Korean J Gastroenterol 2018; 71: 143-152

[33] Spechler S]. Cardiac mucosa: the heart of the problem. Gut 2015; 64: $1673-1674$

[34] Hatakeyama M. Helicobacter pylori CagA and gastric cancer: a paradigm for hit-and-run carcinogenesis. Cell Host Microbe 2014; 15: 306-316 